\title{
Predictors of Insulin Initiation in Patients with Type 2 Diabetes: An Analysis of the Look AHEAD Randomized Trial
}

\author{
Scott J. Pilla, MD, $M H S^{7}$, Hsin-Chieh Yeh, $P h D^{1,2,3}$, Stephen P. Juraschek, $M D, P h D^{7}$, \\ Jeanne M. Clark, MD, MPH ${ }^{1,2,3}$, and Nisa M. Maruthur, MD, $\mathrm{MHS}^{1,2,3}$ \\ 'Department of Medicine, Division of General Internal Medicine, The Johns Hopkins University School of Medicine, Baltimore, MD, USA; \\ ${ }^{2}$ Department of Epidemiology, The Johns Hopkins Bloomberg School of Public Health, Baltimore, MD, USA; ${ }^{3}$ Welch Center for Prevention, \\ Epidemiology \& Clinical Research, Baltimore, MD, USA.
}

BACKGROUND: The decision to initiate insulin in patients with type 2 diabetes is a challenging escalation of care that requires an individualized approach. However, the sociodemographic and clinical factors affecting insulin initiation are not well understood.

OBJECTIVE: We sought to identify patient factors that were independent predictors of insulin initiation among participants in the Look AHEAD (Action for Health in Diabetes) clinical trial.

DESIGN: Retrospective analysis of a randomized clinical trial.

PARTICIPANTS: Beginning in 2001, Look AHEAD enrolled ambulatory U.S. adults with type 2 diabetes who were overweight or obese and had a primary healthcare provider. Participants were randomized (1:1) to an intensive lifestyle intervention, or diabetes support and education. This study examined 3913 participants across the two trial arms who were not using insulin at baseline.

MAIN MEASURES: We used Cox proportional hazards models to estimate the association between participant characteristics and time to insulin initiation. We performed time-varying adjustment for HbAlc measured eight times over the 10-year study period, as well as for multiple clinical and socioeconomic factors.

KEY RESULTS: A total of 1087 participants (27.8\%) initiated insulin during a median follow-up of 8.0 years. Age was inversely associated with insulin initiation (adjusted hazard ratio [aHR] 0.88 per 10 years, $P=0.025)$. The risk of insulin initiation was greater with a higher number of diabetes complications $(P<0.001$ for trend); chronic kidney disease and cardiovascular disease were independently associated with insulin initiation. There was a lower risk of insulin initiation in black (aHR 0.77, $P=0.008$ ) and Hispanic participants (aHR 0.66, $P<0.001$ ) relative to white participants. Socioeconomic factors were not associated with insulin initiation.

\section{NIH Trial Registration Number}

Look AHEAD (Action for Health in Diabetes) trial: NCT00017953

Electronic supplementary material The online version of this article (https://doi.org/10.1007/s11606-017-4282-9) contains supplementary material, which is available to authorized users.

Received August 30, 2017

Revised December 16, 2017

Accepted December 24, 2017

Published online January 19, 2018
CONCLUSIONS: Patient age, race/ethnicity, and diabetes complications may influence insulin initiation in type 2 diabetes, independent of glycemic control. Future work is needed to understand the drivers of racial differences in antihyperglycemic treatment, and to identify patients who benefit most from insulin.

KEY WORDS: diabetes mellitus, type 2; insulin/therapeutic use; risk factors; pharmacoepidemiology.

J Gen Intern Med 33(6):839-46

DOI: $10.1007 / \mathrm{s} 11606-017-4282-9$

๑) Society of General Internal Medicine 2018

\section{INTRODUCTION}

An ongoing challenge in the management of type 2 diabetes is to provide individualized treatment of hyperglycemia in terms of selection of medications and glycemic targets. ${ }^{1}$ Guidelines from the American Diabetes Association and European Association for the Study of Diabetes recommend insulin as one of several second-line antihyperglycemic options after metformin, or first-line therapy with hemoglobin A1c (HbAlc) $\geq 10 \%$. ${ }^{1}$ Insulin is commonly used in the treatment of type 2 diabetes, prescribed in over one fourth of ambulatory visits for type 2 diabetes in the U.S. in 2012. ${ }^{2}$ Although insulin may be more effective than other antihyperglycemic medications in lowering blood glucose, disadvantages include high cost, the need for injections, and increased risk of weight gain and hypoglycemia. ${ }^{1}$

Insulin initiation is an important transition of care from the perspective of both providers and patients. ${ }^{3}$ Preventing insulin initiation is a strong preference and major motivating factor among patients with type 2 diabetes, ${ }^{4,5}$ and primary care physicians list insulin initiation as one of the most difficult aspects of diabetes care. ${ }^{6}$ Patient-reported barriers to insulin use include personal beliefs, difficulty of administration, and cost of therapy. ${ }^{3}$ Due to provider and patient factors, insulin is not used in many patients with significant hyperglycemia. ${ }^{7,8}$

Guidelines suggest that insulin use should be individualized, but do not indicate the criteria for individualization. ${ }^{1}$ Accordingly, providers treating patients with type 2 diabetes have reported varying opinions about the risks and benefits of insulin and the circumstances under which it should be 
started. ${ }^{6}$ While some providers note that comorbid conditions and the degree of hyperglycemia are considerations for starting insulin, ${ }^{9}$ the extent to which these factors are important in practice is not known. In addition, racial/ethnic minorities and patients of lower socioeconomic status have higher HbAlc levels, ${ }^{10-12}$ yet the extent to which antihyperglycemic therapy differs among these populations is not known. Therefore, there is a need to determine the factors that are driving or impeding the use of insulin for patients with type 2 diabetes in clinical practice.

In this study, we use longitudinal data from the Look AHEAD (Action for Health in Diabetes) trial to identify the patient characteristics that are independent predictors of insulin initiation over 10 years of follow-up.

\section{METHODS}

\section{Study Population}

The Look AHEAD trial enrolled 5145 overweight/obese adults with type 2 diabetes from 16 U.S. study centers beginning in 2001. We used the distributed dataset which excludes participants from the Southwest Native American study sites due to consent limitations, resulting in 4901 participants. We examined the 3913 participants who were not using insulin at baseline and had at least one subsequent visit at which medications were recorded. Complete descriptions have been published of the Look AHEAD trial design, recruitment, sample size determination, and outcomes. ${ }^{13,14}$ Look AHEAD eligibility criteria included age $45-76$ years, body mass index (BMI) $\geq 25 \mathrm{~kg} / \mathrm{m}^{2}$ in participants not taking insulin, $\mathrm{HbAlc}<11 \%$, having a primary healthcare provider, and having the ability to complete a maximal exercise test. ${ }^{14}$ Look AHEAD exclusion criteria included recent or exercise-limiting cardiovascular disease, serum creatinine $>1.4 \mathrm{mg} / \mathrm{dL}$ (women) or $1.5 \mathrm{mg} / \mathrm{dL}$ (men), 4+ proteinuria, and need for dialysis. ${ }^{14}$

Participants were randomized 1:1 to the intensive lifestyle intervention (ILI) arm or the diabetes support and education (DSE) arm, which is the control group. ${ }^{15,16}$ The ILI arm received behavioral, nutritional, and pharmacologic interventions aimed at achieving weight loss of $7 \%$ of initial body weight. ${ }^{15}$ The DSE arm received information on diet and physical activity, as well as some social support during group sessions. ${ }^{16}$ Trial staff assessing outcomes were masked to the intervention assignment; participants and interventionists were not masked. Participants in the DSE arm received all diabetes care from their personal healthcare providers; the ILI arm received some monitoring and temporary adjustment of antihyperglycemic medications to reduce the risk of hypoglycemia. ${ }^{14,15}$ The Look AHEAD primary outcome was time to the occurrence of cardiovascular death, non-fatal myocardial infarction, or non-fatal stroke. ${ }^{14}$ The Look AHEAD intervention was terminated in September 2012 due to futility with regard to the primary outcome after a median follow-up of
9.6 years; participants continue to be followed. ${ }^{13}$ This study was administratively censored at 10 years of follow-up.

All Look AHEAD sites received local institutional review board approval, and written informed consent was obtained from all participants. Look AHEAD is registered with ClinicalTrials.gov (NCT00017953). The analyses performed for this study were not conducted at the Look AHEAD Data Coordinating Center, and this is not the work of the Look AHEAD study group.

\section{Study Outcome}

The primary outcome of this study was time to first use of any insulin medication. Insulin use was ascertained by a medication inventory form completed by trained trial staff at yearly study visits. Participants were asked to bring their home medications to each visit for review, and were asked specifically about insulin use. Time to event was defined as the length of time from the date of the baseline medication inventory form to the date at which the first use of insulin was reported. Participants with gaps in medication data were excluded from analysis during gap periods, and did not accrue time at risk during those periods.

\section{Predictor Variables}

Sociodemographic predictors were ascertained by selfadministered questionnaire and analyzed as time-fixed from baseline values. Blood pressure and anthropometry were measured yearly by standardized protocols. ${ }^{14}$ Hypertension was defined as systolic blood pressure $>140 \mathrm{mmHg}$, diastolic blood pressure $>90 \mathrm{mmHg}$, or use of antihypertensive medications. Dyslipidemia was defined as LDL $>130 \mathrm{mg} / \mathrm{dL}$ or use of lipid-lowering medications. Diabetic neuropathy and retinopathy were ascertained by questionnaire. Hypertension, dyslipidemia, diabetic neuropathy, and diabetic retinopathy were analyzed as time-varying in a non-recurrent manner (i.e. once hypertensive, then always hypertensive). Cardiovascular disease was assessed at baseline and included selfreported history of myocardial infarction, stroke, angina, heart failure, prior cardiac bypass surgery, coronary stenting, carotid endarterectomy, or peripheral vascular angioplasty. The number of antihyperglycemic drugs was categorized to create three groups of approximately equal size throughout the study period, and analyzed as time-varying.

Laboratory measurements were performed yearly from baseline to year 4 , and in alternating years thereafter. $\mathrm{HbAlc}$ was analyzed as time-varying, and was offset by one study visit such that the HbAlc value at the study visit prior to the outcome was used as the predictor. This was done because the $\mathrm{HbAlc}$ value prior to insulin treatment (as opposed to after insulin treatment was started) was considered to be the most clinically relevant to the initiation of insulin. Chronic kidney disease was analyzed in a non-recurrent manner and defined according to the Kidney Disease: Improving Global Outcomes (KDIGO) 2012 guidelines ${ }^{17}$ as estimated glomerular filtration 
rate (eGFR) by the Chronic Kidney Disease Epidemiology Collaboration (CKD-Epi) equation ${ }^{18}<60 \mathrm{~mL} / \mathrm{min} / 1.73 \mathrm{~m}^{2}$, or urine albumin creatinine ratio $\geq 30 \mathrm{mg} / \mathrm{g}$. For analysis of the association between insulin initiation and number of diabetes complications, diabetes complications included cardiovascular disease, chronic kidney disease, diabetic neuropathy, and diabetic retinopathy.

All predictor variables were missing less than $3 \%$ of baseline observations, except for yearly family income, which was missing $10 \%$ of baseline observations; therefore, a subgroup was added for participants with missing family income data. Missing longitudinal data in timevarying predictor variables was carried forward from the prior observation.

\section{Statistical Analysis}

Baseline characteristics were described via means and proportions, and compared across categories of race/ethnicity using one-way analysis of variance for continuous variables or $\chi^{2}$ tests for categorical variables. The jackknife method was used to calculate confidence intervals for incidence rates.

The association between participant characteristics and insulin initiation was assessed using three Cox proportional hazards models: adjusted for the Look AHEAD treatment arm only (model 1); adjusted for age, gender, race/ethnicity, HbAlc, and treatment arm (model 2); and adjusted for all predictors (model 3). Predictors in model 2 were selected a priori to adjust for basic demographics and $\mathrm{HbAlc}$, which was considered a potential major confounder; model 3 added all predictors. No violations of the proportional hazards assumption were found by examining unadjusted and adjusted (per model 3) $\log -\log$ survival curves, with continuous predictors categorized by quartile.

To examine potential nonlinear associations between continuous predictors and the outcome, we constructed restricted cubic spline models with hazard ratios expressed relative to the median of the predictor, with four knots determined by Harrell's method. ${ }^{19}$ Cubic spline plots were truncated at the 0.5 th and 99.5 th percentiles of the distribution of the predictor.

Two-sided $P \leq 0.05$ was considered statistically significant. Analyses were performed using STATA version 14 software (StataCorp LP, College Station, TX).

\section{Sensitivity Analyses}

We performed sensitivity analyses to determine the impact of covariate adjustment and selection of the analytic population. To maximize the ability of our model to detect significant associations, we reduced the number of covariates in model 3 using forward and backward stepwise selection by $P<0.15$ and by the minimum Akaike information criterion (AIC). All selection methods yielded similar results; forward selection for minimum AIC is presented. To determine whether inclusion of participants randomized to the ILI arm affected the observed associations, we performed the fully adjusted analysis restricted to only the participants in the DSE arm $(n=1941)$.

\section{RESULTS}

\section{Participant Characteristics}

The baseline characteristics of the 3913 participants who met inclusion criteria are shown in Table 1. The mean age was

Table 1 Participant Baseline Characteristics

\begin{tabular}{|c|c|}
\hline Characteristic & Value \\
\hline$N$ & 3913 \\
\hline Randomized to the ILI arm & $1966(50.2)$ \\
\hline Age (years) & $58.9 \pm 6.7$ \\
\hline Female & $2300(58.8)$ \\
\hline \multicolumn{2}{|l|}{ Race/ethnicity } \\
\hline White, non-Hispanic & $2658(67.9)$ \\
\hline Black, non-Hispanic & $613(15.7)$ \\
\hline Hispanic & $505(12.9)$ \\
\hline Other & $137(3.5)$ \\
\hline HbA1c (\%) & $7.1 \pm 1.1$ \\
\hline \multicolumn{2}{|l|}{ Smoking status } \\
\hline Never & 1947 (49.9) \\
\hline Former & $1803(46.2)$ \\
\hline Current & $154(3.9)$ \\
\hline BMI $\left(\mathrm{kg} / \mathrm{m}^{2}\right)$ & $35.7 \pm 5.9$ \\
\hline Hypertension & $3238(82.8)$ \\
\hline Antihypertensive medication use & $2825(72.3)$ \\
\hline Dyslipidemia & $2718(69.5)$ \\
\hline Lipid lowering medication use & 1950 (49.9) \\
\hline Chronic kidney disease & 689 (17.6) \\
\hline Cardiovascular disease & $488(12.5)$ \\
\hline Diabetic neuropathy & $388(9.9)$ \\
\hline Diabetic retinopathy & $216(5.5)$ \\
\hline Diabetes duration (years) & $5.5 \pm 5.4$ \\
\hline Family history of diabetes & $2472(63.2)$ \\
\hline \multicolumn{2}{|l|}{ Source of medical care } \\
\hline Private office & $3059(78.3)$ \\
\hline Hospital-affiliated clinic & $363(9.3)$ \\
\hline Community care center & $246(6.3)$ \\
\hline Other & $218(5.6)$ \\
\hline No usual care & $19(0.5)$ \\
\hline No medical insurance & $290(7.4)$ \\
\hline Unemployed & $789(20.2)$ \\
\hline \multicolumn{2}{|l|}{ Highest level of education } \\
\hline Doctorate or professional degree & $207(5.3)$ \\
\hline Bachelor's or master's degree & $1475(37.7)$ \\
\hline High school diploma & $1928(49.3)$ \\
\hline Less than high school & $221(5.7)$ \\
\hline Other & $81(2.1)$ \\
\hline \multicolumn{2}{|l|}{ Yearly family income } \\
\hline$>\$ 80,000$ & $1100(28.1)$ \\
\hline$\$ 60,000-80,000$ & $595(15.2)$ \\
\hline$\$ 40,000-60,000$ & $731(18.7)$ \\
\hline$\$ 20,000-40,000$ & $720(18.4)$ \\
\hline$<\$ 20,000$ & $371(9.5)$ \\
\hline Missing & $396(10.1)$ \\
\hline \multicolumn{2}{|l|}{ No. of antihyperglycemic drugs } \\
\hline $0-1$ & $2324(60.0)$ \\
\hline 2 & $1212(31.4)$ \\
\hline 3 or more & $335(8.7)$ \\
\hline Metformin use & $2389(62.2)$ \\
\hline Sulfonylurea use & $1917(50.3)$ \\
\hline Thiazolidinedione use & $969(25.5)$ \\
\hline Meglitinide use & $119(3.2)$ \\
\hline Alpha-glucosidase inhibitor use & $28(0.8)$ \\
\hline
\end{tabular}

Data are mean \pm standard deviation or frequency (\%). ILI, intensive lifestyle intervention 
58.9 years; $58.8 \%$ were female; $67.9 \%, 15.7 \%, 12.9 \%$ and $3.5 \%$ were white, black, Hispanic, and other races, respectively. The mean HbA1c was $7.1 \%$, and the mean duration of diabetes was 5.5 years. Participants were under observation for a median of 8.0 years, accruing 26,870 person-years at risk.

\section{Absolute Rates of Insulin Initiation}

During the study period, 1087 participants (27.8\%) initiated insulin, with an overall incidence rate of 4.05 events per 100 person-years (95\% CI 3.83-4.28). Among the 418 participants with baseline HbA1c >8.5\%, 203 (48.6\%) initiated insulin during the study period, with an incidence rate of 8.43 events per 100 person-years (95\% CI 7.43-9.58). The mean HbAlc at the study visit prior to insulin initiation was $8.1 \pm 1.6 \%$.

\section{Relative Hazards of Insulin Initiation}

Table 2 shows adjusted hazard ratios (HR) for insulin initiation using three analytic models. In all models, age was inversely associated with insulin initiation, with a fully adjusted HR of 0.88 per 10 years $(95 \%$ CI $0.79-0.98, P=0.025)$. There was no difference in the risk of insulin initiation by gender in the fully adjusted analysis. Black and Hispanic race/ethnicity were

Table 2 Hazard Ratios for Insulin Initiation by Participant Characteristics

\begin{tabular}{|c|c|c|c|}
\hline Characteristic & $\begin{array}{l}\text { HR }(95 \% \text { CI) } \\
\text { Model 1* }\end{array}$ & $\begin{array}{l}\text { HR (95\% CI) } \\
\text { Model } 2^{\dagger}\end{array}$ & $\begin{array}{l}\text { HR (95\% CI) } \\
\text { Model } 3^{\ddagger}\end{array}$ \\
\hline Age (per 10 years) & $0.77(0.71-0.85)$ & $0.89(0.81-0.98)$ & $0.88(0.79-0.98)$ \\
\hline Female & $0.88(0.78-1.00)$ & $0.88(0.78-1.00)$ & $1.10(0.95-1.26)$ \\
\hline Race/ethnicity & $P=0.004$ & $\mathrm{P}<0.001$ & $\mathrm{P}<0.001$ \\
\hline White, non-Hispanic & Reference & Reference & Reference \\
\hline Black, non-Hispanic & $0.78(0.65-0.93)$ & $0.62(0.51-0.74)$ & $0.77(0.63-0.93)$ \\
\hline Hispanic & $0.78(0.64-0.94)$ & $0.60(0.49-0.72)$ & $0.66(0.52-0.83)$ \\
\hline Other & $1.08(0.80-1.47)$ & $1.04(0.77-1.42)$ & $1.08(0.79-1.48)$ \\
\hline $\mathrm{HbAlc}($ per $1 \%)$ & $1.48(1.44-1.52)$ & $1.50(1.46-1.55)$ & $1.40(1.36-1.45)$ \\
\hline Smoking status & $P<0.001$ & $P<0.001$ & $P=0.003$ \\
\hline Never & Reference & Reference & Reference \\
\hline Former & $1.06(0.94-1.20)$ & $1.12(0.99-1.27)$ & $1.09(0.96-1.24)$ \\
\hline Current & $1.72(1.31-2.26)$ & $1.69(1.28-2.22)$ & $1.62(1.22-2.16)$ \\
\hline BMI (per $5 \mathrm{~kg} / \mathrm{m}^{2}$ ) & $1.17(1.12-1.22)$ & $1.17(1.12-1.23)$ & $1.05(1.00-1.11)$ \\
\hline Hypertension & $1.90(1.41-2.58)$ & $2.20(1.62-2.98)$ & $1.46(1.07-2.00)$ \\
\hline Dyslipidemia & $1.33(1.07-1.65)$ & $1.33(1.07-1.65)$ & $1.10(0.88-1.38)$ \\
\hline Chronic kidney disease & $1.44(1.27-1.62)$ & $1.28(1.13-1.45)$ & $1.17(1.03-1.33)$ \\
\hline Cardiovascular disease & $1.49(1.27-1.76)$ & $1.62(1.37-1.93)$ & $1.56(1.31-1.86)$ \\
\hline Diabetic neuropathy & $1.37(1.20-1.55)$ & $1.34(1.18-1.52)$ & $1.07(0.94-1.22)$ \\
\hline Diabetic retinopathy & $1.36(1.17-1.59)$ & $1.21(1.04-1.42)$ & $1.02(0.87-1.20)$ \\
\hline Diabetes duration (per 10 years) & $1.47(1.35-1.60)$ & $1.49(1.36-1.64)$ & $1.10(0.98-1.23)$ \\
\hline No. of antihyperglycemic drugs & $P<0.001$ & $P<0.001$ & $P<0.001$ \\
\hline $0-1$ & Reference & Reference & Reference \\
\hline 2 & $5.66(4.51-7.08)$ & $4.47(3.57-5.60)$ & $4.12(3.28-5.18)$ \\
\hline 3 or more & $21.65(17.46-26.82)$ & $15.64(12.58-19.45)$ & 14.01(11.21-17.51) \\
\hline Family history of diabetes & $1.18(1.04-1.34)$ & $1.22(1.07-1.39)$ & $1.15(1.01-1.31)$ \\
\hline Source of medical care & $P<0.001$ & $P<0.001$ & $P<0.001$ \\
\hline Private office & Reference & Reference & Reference \\
\hline Hospital-affiliated clinic & $1.40(1.17-1.68)$ & $1.40(1.16-1.69)$ & $1.49(1.23-1.81)$ \\
\hline Community care center & $0.79(0.55-1.13)$ & $0.70(0.49-1.02)$ & $0.76(0.51-1.11)$ \\
\hline Other & $1.47(1.11-1.95)$ & $1.35(1.02-1.79)$ & $1.60(1.21-2.13)$ \\
\hline No usual care & $0.17(0.02-1.19)$ & $0.12(0.02-0.85)$ & $0.33(0.05-2.37)$ \\
\hline No health insurance & $0.99(0.78-1.26)$ & $0.94(0.73-1.21)$ & $1.11(0.84-1.47)$ \\
\hline Unemployed & $1.02(0.89-1.17)$ & $1.13(0.98-1.29)$ & $1.10(0.96-1.27)$ \\
\hline Highest level of education ${ }^{\S}$ & $P=0.786$ & $P=0.226$ & $P=0.408$ \\
\hline Doctorate or professional degree & Reference & Reference & Reference \\
\hline Bachelor's or master's degree & $1.06(0.80-1.40)$ & $1.19(0.90-1.58)$ & $1.24(0.93-1.65)$ \\
\hline High school diploma & $1.11(0.84-1.47)$ & $1.27(0.96-1.69)$ & $1.28(0.96-1.71)$ \\
\hline Less than high school & $0.92(0.63-1.35)$ & $1.06(0.70-1.61)$ & $1.02(0.66-1.58)$ \\
\hline Other & $0.96(0.58-1.57)$ & $1.23(0.75-2.03)$ & $1.37(0.83-2.27)$ \\
\hline Yearly family income ${ }^{\S}$ & $P=0.011$ & $P=0.112$ & $P=0.541$ \\
\hline$>\$ 80,000$ & Reference & Reference & Reference \\
\hline$\$ 60,000-80,000$ & $1.05(0.87-1.25)$ & $1.13(0.94-1.35)$ & $0.99(0.82-1.19)$ \\
\hline$\$ 40,000-60,000$ & $0.86(0.72-1.03)$ & $0.84(0.70-1.02)$ & $0.85(0.70-1.03)$ \\
\hline$\$ 20,000-40,000$ & $0.83(0.69-1.00)$ & $0.91(0.74-1.10)$ & $0.96(0.77-1.18)$ \\
\hline$<\$ 20,000$ & $0.84(0.66-1.06)$ & $0.92(0.71-1.19)$ & $0.96(0.72-1.28)$ \\
\hline Missing & $0.97(0.78-1.20)$ & $1.13(0.90-1.41)$ & $1.21(0.96-1.53)$ \\
\hline Randomized to the ILI arm & $0.73(0.65-0.82)$ & $0.79(0.70-0.89)$ & $0.96(0.85-1.08)$ \\
\hline
\end{tabular}

For multi-categorical variables, the global P-value (nominal variables) or P-value for linear trend (ordinal variables) is displayed on the row aligned with the variable name. Boldface type indicates $P \leq 0.05$. ILI, intensive lifestyle intervention

* Each characteristic is adjusted for treatment arm only

tEach characteristic is adjusted for age, gender, racelethnicity, HbAlc, and treatment arm

$¥$ Each characteristic is adjusted for all listed characteristics

$\S$ The "Other" and "Missing" categories were omitted from analysis of linear trends 
associated with a significantly lower risk of insulin initiation relative to white race in all models. Details of baseline characteristics stratified by race/ethnicity may be found in Online Supplemental Table S1.

Figure 1 shows the fully adjusted HR for insulin initiation (vertical axis) across the range of each continuous characteristic (age, HbA1c, BMI, and diabetes duration - horizontal axis) using a restricted cubic spline model to capture nonlinear relationships; the distribution of the characteristic among participants is shown in the lower part of the plot.

In the fully adjusted analysis (Table 2), HbA1c was positively associated with insulin initiation (HR 1.40 per $1 \%$, $P<0.001$ ), as was the number of antihyperglycemic drugs (global $P<0.001$ ). Additional clinical factors independently associated with insulin initiation were BMI (HR 1.05 per $5 \mathrm{~kg} /$ $\mathrm{m}^{2}, P=0.036$ ), smoking status (global $P=0.003$ ), hypertension (HR 1.46, $P=0.017$ ), chronic kidney disease (HR 1.17, $P=0.015$ ), cardiovascular disease (HR 1.56, $P<0.001$ ), and a family history of diabetes (HR 1.15, $P=0.034$ ).

The fully adjusted association between number of diabetes complications (cardiovascular disease, chronic kidney disease, diabetic neuropathy, and diabetic retinopathy) and insulin initiation is shown in Figure 2. Insulin initiation was positively associated with number of complications, with an HR of 1.16 (95\% CI 1.08-1.24) per each additional complication $(P<0.001$ for trend). a

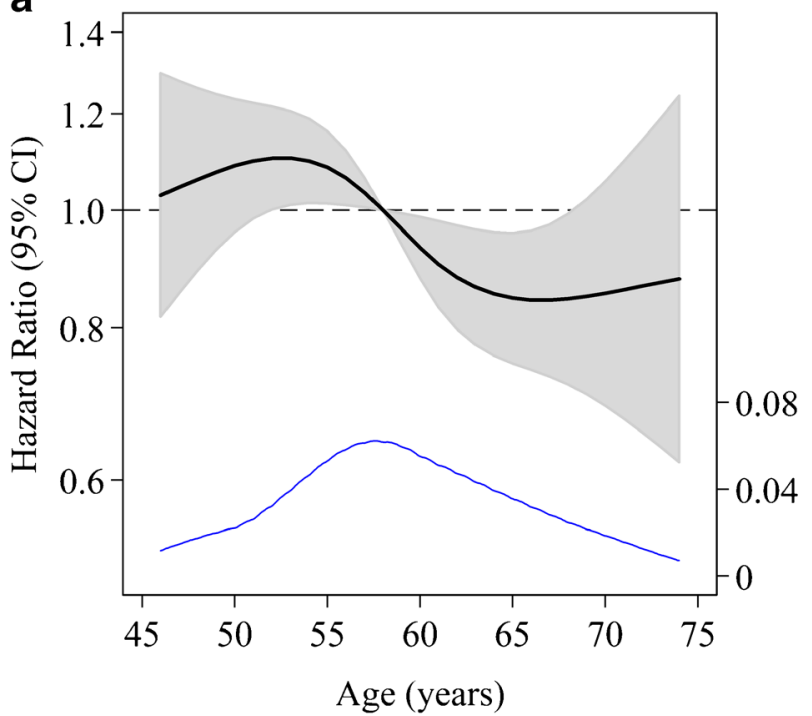

C

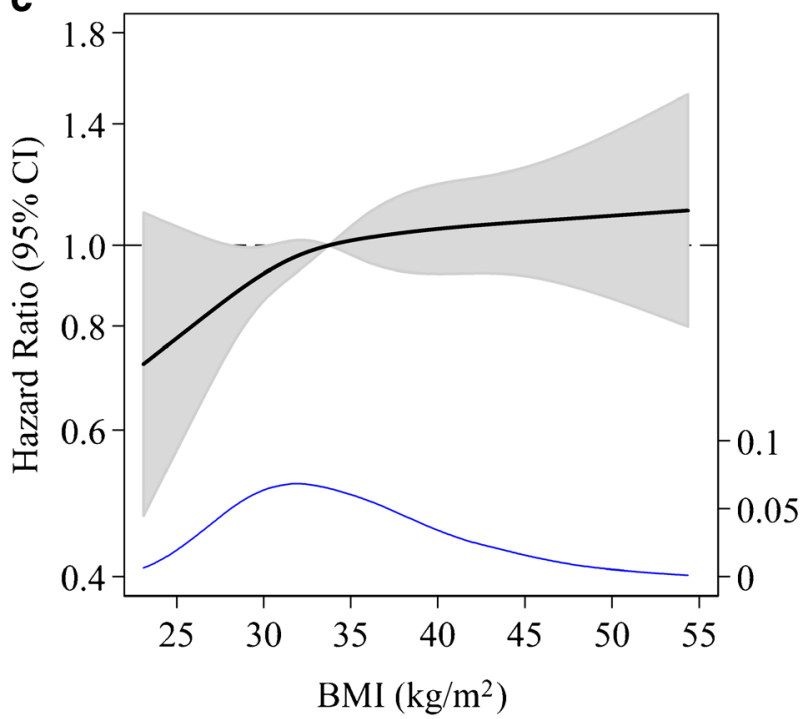

b

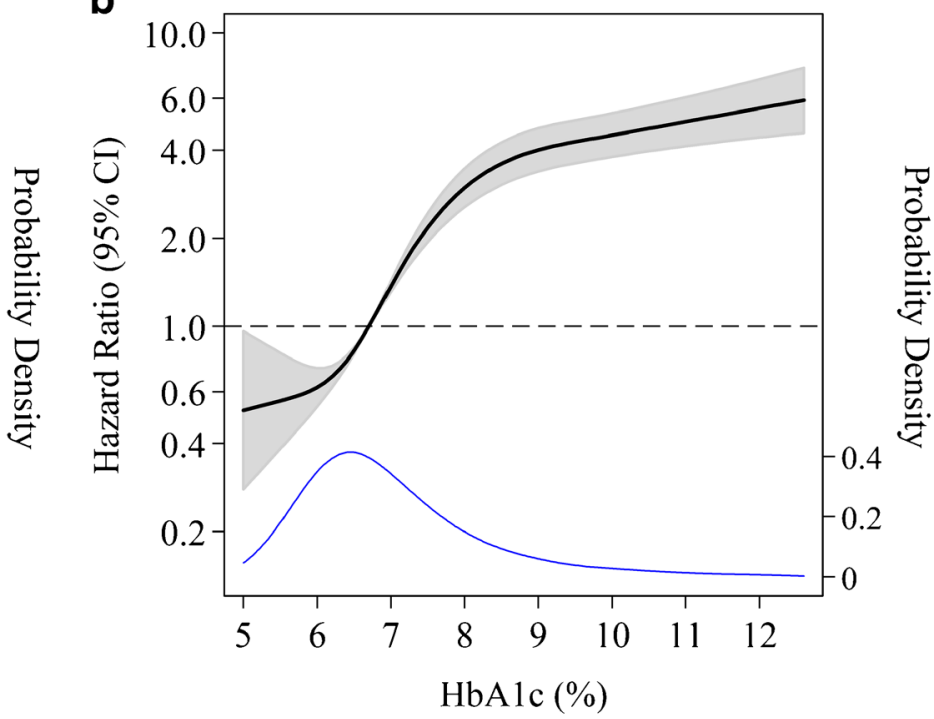

d

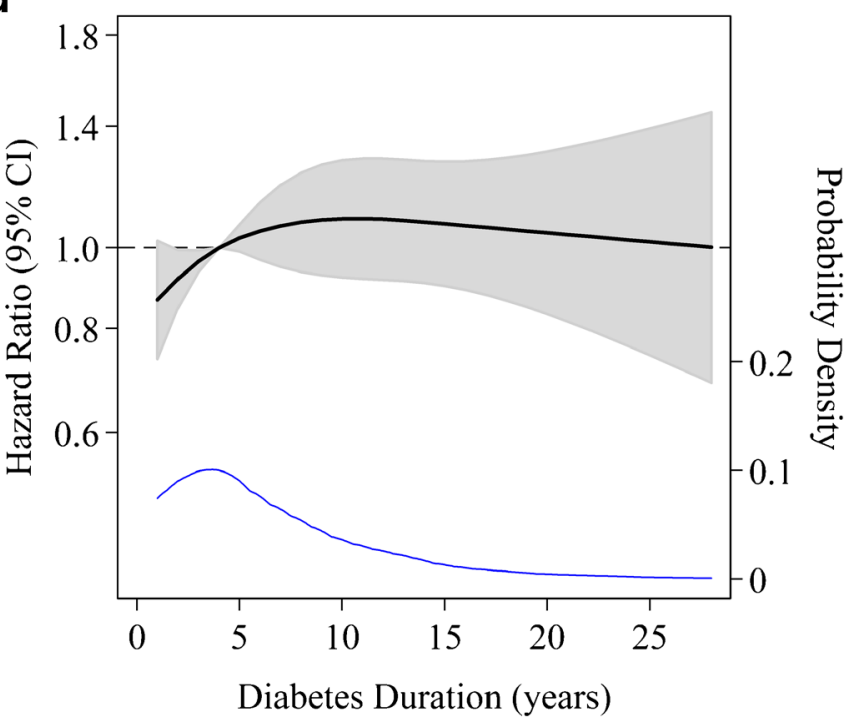

Figure 1 Adjusted hazard ratios for insulin initiation by continuous patient characteristics using restricted cubic spline models. Continuous predictors are baseline age (Panel A), hemoglobin A1c (HbA1c) (Panel B), body mass index (BMI) (Panel C), and baseline diabetes duration (Panel D). Hazard ratios (displayed as heavy black lines) are expressed relative to the median of the predictor, with four knots determined by Harrell's method; ${ }^{19}$ gray shaded areas represent $95 \%$ confidence intervals. Kernel density plots depicting the distribution of the predictor are overlaid (thin blue lines), with probability density labeled on the right axis; plots are truncated at the 0.5 th and 99.5 th percentiles of the distribution of the predictor. All hazard ratios are fully adjusted (model 3 ). 


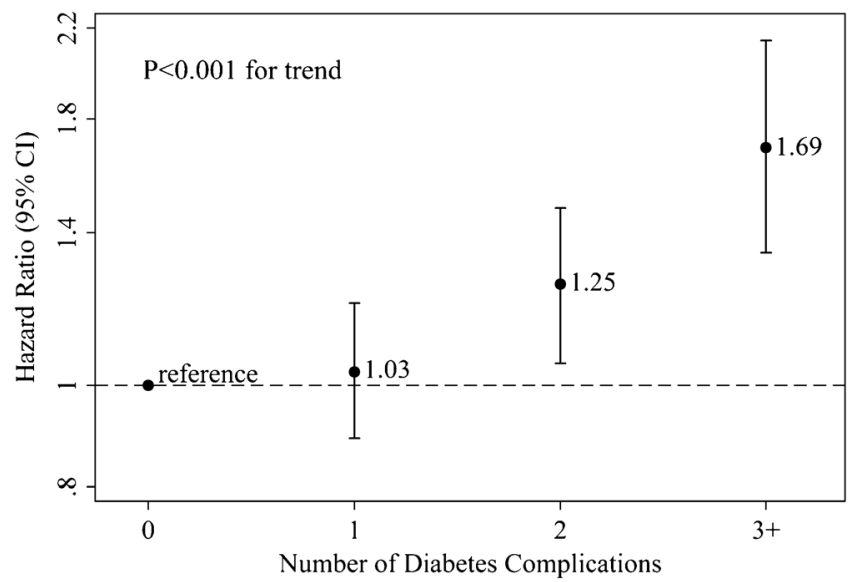

Figure 2 Adjusted hazard ratios for insulin initiation by number of diabetes complications. Diabetes complications included in this analysis are cardiovascular disease, chronic kidney disease, diabetic neuropathy, and diabetic retinopathy. Hazard ratios are fully adjusted (model 3) except for the specified diabetes complications. 95\% Confidence intervals for the hazard ratios of 1,2 , and $3+$ diabetes complications are (0.89-1.20), (1.05-1.48), and (1.34-2.14), respectively.

In addition, source of medical care was associated with insulin initiation in the fully adjusted model (global $P<0.001)$, with an HR of 1.49 (95\% CI 1.23-1.81) for the subgroup receiving care at a hospital-affiliated clinic relative to care at a private office. No characteristic related to socioeconomic status was associated with insulin initiation in the fully adjusted model.

As has been shown previously, ${ }^{20}$ participants randomized to the Look AHEAD intensive lifestyle intervention had a significantly lower risk of insulin initiation than those in the DSE arm in model 1.

Results of sensitivity analyses for covariate selection and inclusion of the ILI study arm can be found in the online supplemental material.

\section{DISCUSSION}

In this study, we identified multiple independent predictors of insulin initiation in overweight and obese adults with type 2 diabetes. Insulin initiation was inversely associated with older age and black or Hispanic race/ethnicity, and was positively associated with $\mathrm{HbAlc}$, current smoking, BMI, hypertension, the presence macrovascular and microvascular complications, number of antihyperglycemic drugs, family history of diabetes, and care at a hospital-affiliated medical clinic. While glycemic control was strongly associated with insulin initiation, insulin was started in less than $10 \%$ of participants per year with baseline $\mathrm{HbA} 1 \mathrm{c}>8.5 \%$. These findings suggest that only a minority of patients with higher $\mathrm{HbAlc}$ values are initiating insulin each year, and factors beyond the degree of hyperglycemia influence whether insulin treatment is started.

Notably, insulin initiation in this study does not necessarily indicate optimal or suboptimal care, but rather treatment differences that may reflect clinical practice, patient preference, or both. By adjusting for HbAlc and other characteristics, we were able to explore factors that may be independently driving or impeding insulin initiation outside of glycemic control. To our knowledge, this is the first study to examine the relationship between socioeconomic factors and insulin initiation, and the first to include time-varying adjustment for $\mathrm{HbAlc}$ and antihyperglycemic treatment intensity to account for longitudinal changes in diabetes features and therapy which may confound the association between patient characteristics and insulin initiation.

In this study, we found that age was inversely associated with insulin initiation, with a significantly lower risk primarily in participants aged 60 years and older. This finding is in agreement with results of prior studies. ${ }^{21-27}$ It is possible that this finding reflects provider practice, i.e. choosing to limit the use of insulin in older patients. It is also possible that older adults are less likely than younger patients to assent to insulin treatment. ${ }^{3}$ Further study is needed to explain this.

Patients in this study with a greater number of diabetes complications were more likely to initiate insulin. The few prior studies examining this have also found that insulin initiation is associated with microvascular and macrovascular complications, ${ }^{24,25}$ or a higher Charlson comorbidity index. ${ }^{23}$ These findings suggest that providers are preferentially initiating insulin in patients with a higher burden of diabetes complications, that such patients are more receptive to insulin use, or both. Insulin initiation may be associated with chronic kidney disease because of contraindications to other medications in the setting of reduced renal function. ${ }^{1}$ There is little evidence to inform whether insulin versus other antihyperglycemic medications should be used in patients with established complications, but guidelines suggest a higher HbAlc target in these patients. ${ }^{1}$ In addition, we found that BMI was positively associated with insulin initiation, which has also been found in previous studies, ${ }^{23,25}$ although the reasons for this are unclear. It will be important to determine why patients with a higher BMI may be preferentially initiating insulin, as insulin use can exacerbate weight gain. ${ }^{1}$

We found a lower risk of insulin initiation in black and Hispanic than white participants, independent of their socioeconomic status. However, racial/ethnic disparities in living and working conditions are not entirely captured by socioeconomic measures, which could result in residual confounding. ${ }^{28}$ In addition, a greater proportion of minority participants $(22.3 \%)$ than white participants $(16.6 \%)$ were excluded for baseline insulin use, which may have introduced bias if retained minority participants were at a lower risk of insulin initiation. We believe that this is less likely, as black and Hispanic participants had higher HbAlc values and no difference in diabetes duration or number of antihyperglycemic medications at baseline (Online Supplemental Table S1). A prior analysis of Look AHEAD baseline data, ${ }^{29}$ along with other studies, ${ }^{30}$ have found that racial/ethnic minorities with type 2 diabetes experience suboptimal control of hyperglycemia and cardiovascular risk factors. The results of the present 
study provide evidence of racial differences in insulin initiation. Further research is needed to examine the provider and patient factors contributing to differential treatment of patients with type 2 diabetes by race/ethnicity, and to determine whether these differences are driving suboptimal glycemic control.

In this study of a primarily medically insured population, we found no clear association between markers of socioeconomic status and insulin initiation. As patients have reported that cost of insulin is a barrier to treatment, ${ }^{3}$ further study is needed to determine whether socioeconomic factors are impacting insulin utilization, particularly in uninsured or otherwise disadvantaged populations.

The major strength of this study is that participants were characterized by standardized protocols and closely followed for up to 10 years. This allowed us to control for $\mathrm{HbAlc}$ and other potential confounders in a time-dependent manner with a high degree of granularity. In addition, we performed multiple analytic models and sensitivity analyses through which our findings were robust. However, this study has several limitations. The transition to insulin therapy involves both provider practice and patient preference, and this study is not able to distinguish between these factors. HbAlc was measured at annual or biennial intervals, so the exact $\mathrm{HbAlc}$ at the time of insulin initiation is not known. Additionally, the ascertainment of neuropathy and retinopathy was by self-report, which is less valid than objective measures.

Participants were recruited as outpatients from 16 study sites throughout the U.S., thus representing a diverse sample of ambulatory U.S. adults with type 2 diabetes. As Look AHEAD enrolled only overweight and obese persons, these results may not be applicable to leaner patients with type 2 diabetes. Additionally, patients recruited to clinical trials may be different from the general population in terms of demographics, comorbidities, and medical adherence.

Overall, results of this study show that patient age, race/ethnicity, diabetes complications, and other clinical features were independently associated with insulin initiation in adults with type 2 diabetes. The predictors of insulin initiation identified in this study should guide future efforts to determine the patient and provider contributions to the insulin transition, and to inform evidence-based strategies for tailoring the use of insulin to a patient's characteristics and needs.

Corresponding Author: Scott J. Pilla, MD, MHS; Department of Medicine, Division of General Internal Medicine, The Johns Hopkins University School of Medicine, Baltimore, MD, USA (e-mail: spilla1@jhmi.edu).

Contributors S.J.P performed the data analysis and prepared the manuscript. H.Y. contributed to the methods and reviewed/edited the manuscript. J.M.C. contributed to the study design, data collection, methods, and discussion, and reviewed/edited the manuscript. S.P.J. and N.M.M. contributed to the methods and discussion, and reviewed/edited the manuscript. The authors thank Moyses Szklo, $M D, D r P H$, of the Johns Hopkins Bloomberg School of Public Health for contributions to the design and interpretation of this work. S.J.P. is the guarantor of this work and, as such, had full access to the data and takes responsibility for the accuracy and integrity of this research.
Funders S.J.P. was supported by NIH training grant 5T32HLOO718040 (PI: D. Levine). S.P.J. was supported by NIH/NIDDK grant T32DK007732 (PI: L. Appel). J.M.C. and N.M.M. were supported by NIH/NIDDK grant 2UO1DK057149-17 (PI: J. Clark).

\section{Compliance with Ethical Standards:}

Prior Presentations: Parts of this research were presented as an oral presentation at the Society of General Internal Medicine Annual Meeting on April 21, 2017.

Conflict of Interest: The authors declare that they do not have a conflict of interest.

\section{REFERENCES}

1. American Diabetes Association. 8. Pharmacologic Approaches to Glycemic Treatment. Diabetes Care. 2017;40(Suppl 1):S64-S74.

2. Turner LW, Nartey D, Stafford RS, Singh S, Alexander GC. Ambulatory treatment of type 2 diabetes in the U.S., 1997-2012. Diabetes Care 2014;37(4):985-92.

3. Ng CJ, Lai PS, Lee YK, Azmi SA, Teo CH. Barriers and facilitators to starting insulin in patients with type 2 diabetes: a systematic review. Int $\mathrm{J}$ Clin Pract 2015;69(10): 1050-70.

4. Hayes RP, Bowman L, Monahan PO, Marrero DG, McHorney CA Understanding diabetes medications from the perspective of patients with type 2 diabetes: prerequisite to medication concordance. Diabetes Educ 2006;32(3):404-14.

5. von Arx LB, Kjeer T. The patient perspective of diabetes care: a systematic review of stated preference research. Patient 2014;7(3):283-300.

6. Hayes RP, Fitzgerald JT, Jacober SJ. Primary care physician beliefs about insulin initiation in patients with type 2 diabetes. Int $\mathrm{J}$ Clin Pract 2008;62(6):860-8.

7. Nichols GA, Koo YH, Shah SN. Delay of insulin addition to ora combination therapy despite inadequate glycemic control: delay of insulin therapy. J Gen Intern Med 2007;22(4):453-8.

8. Rubino A, McGuay LJ, Gough SC, Kvasz M, Tennis P. Delayed initiation of subcutaneous insulin therapy after failure of oral glucoselowering agents in patients with Type 2 diabetes: a population-based analysis in the UK. Diabet Med 2007;24(12):1412-8.

9. Grant RW, Wexler DJ, Watson AJ, Lester WT, Cagliero E, Campbell EG, et al. How doctors choose medications to treat type 2 diabetes: a national survey of specialists and academic generalists. Diabetes Care 2007;30(6): 1448-53

10. Kirk JK, D'Agostino RB, Jr., Bell RA, Passmore LV, Bonds DE, Karter AJ, et al. Disparities in HbAlc levels between African-American and nonHispanic white adults with diabetes: a meta-analysis. Diabetes Care 2006;29(9):2130-6.

11. Kirk JK, Passmore LV, Bell RA, Narayan KM, D'Agostino RB Jr, Arcury TA, et al. Disparities in AlC levels between Hispanic and nonHispanic white adults with diabetes: a meta-analysis. Diabetes Care 2008;31(2):240-6.

12. Houle J, Lauzier-Jobin F, Beaulieu MD, Meunier S, Coulombe S, Cote $\mathbf{J}$, et al. Socioeconomic status and glycemic control in adult patients with type 2 diabetes: a mediation analysis. BMJ Open Diabetes Res Care 2016;4(1):e000184.

13. The Look AHEAD Research Group. Cardiovascular effects of intensive lifestyle intervention in type 2 diabetes. N Engl J Med 2013;369(2):145-54.

14. Ryan DH, Espeland MA, Foster GD, Haffner SM, Hubbard VS, Johnson KC, et al. Look AHEAD (Action for Health in Diabetes): design and methods for a clinical trial of weight loss for the prevention of cardiovascular disease in type 2 diabetes. Control Clin Trials 2003;24(5):610-28.

15. The Look AHEAD Research Group, Wadden TA, West DS, Delahanty L, Jakicic J, Rejeski J, et al. The Look AHEAD study: a description of the lifestyle intervention and the evidence supporting it. Obesity (Silver Spring). 2006; 14(5):737-52.

16. Wesche-Thobaben JA. The development and description of the comparison group in the Look AHEAD trial. Clin Trials 2011;8(3):320-9.

17. Kidney Disease: Improving Global Outcomes (KDIGO) Chronic Kidney Disease Work Group. KDIGO clinical practice guideline for the evaluation and management of chronic kidney disease. Kidney Int 2013;3 (suppl 3):1-150. 
18. Levey AS, Stevens LA, Schmid CH, Zhang YL, Castro AF 3rd, Feldman HI, et al. A new equation to estimate glomerular filtration rate. Ann Intern Med 2009;150(9):604-12.

19. Harrell FE, Jr., Lee KL, Pollock BG. Regression models in clinical studies: determining relationships between predictors and response. J Natl Cancer Inst 1988;80(15):1198-202.

20. The Look AHEAD Research Group. Long-term effects of a lifestyle intervention on weight and cardiovascular risk factors in individuals with type 2 diabetes mellitus: four-year results of the Look AHEAD trial. Arch Intern Med 2010;170(17):1566-75.

21. Ringborg A, Lindgren P, Yin DD, Martinell M, Stalhammar J. Time to insulin treatment and factors associated with insulin prescription in Swedish patients with type 2 diabetes. Diabete Metab 2010;36(3):198203.

22. Janghorbani M, Amini M. Predictors of switching to insulin from noninsulin therapy in patients with type 2 diabetes mellitus. Diabetes Res Clin Pract 2011:92(1):111-7.

23. Parchman ML, Wang CP. Initiation of insulin among veterans with type 2 diabetes and sustained elevation of Alc. Prim Care Diabetes 2012;6(1):19-25.

24. Kostev K, Dippel FW, Rathmann W. Predictors of insulin initiation in metformin and sulfonylurea users in primary care practices: the role of kidney function. J Diabetes Sci Technol 2014;8(5):1023-8.
25. Danne T, Bluhmki T, Seufert J, Kaltheuner M, Rathmann W, Beyersmann J, et al. Treatment intensification using long-acting insulin -predictors of future basal insulin supported oral therapy in the DIVE registry. BMC Endocr Disord 2015;15:54.

26. Machado-Alba JE, Machado-Duque ME, Moreno-Gutierrez PA. Time to and factors associated with insulin initiation in patients with type 2 diabetes mellitus. Diabetes Res Clin Pract 2015;107(3):332-7.

27. Mast R, Danielle Jansen AP, Walraven I, Rauh SP, van der Heijden AA, Heine RJ, et al. Time to insulin initiation and long-term effects of initiating insulin in people with type 2 diabetes mellitus: the Hoorn Diabetes Care System Cohort Study. Eur J Endocrinol 2016;174(5):56371.

28. Krieger N, Williams DR, Moss NE. Measuring social class in US public health research: concepts, methodologies, and guidelines. Annu Rev Public Health 1997; 18:341-78.

29. Bertoni AG, Clark JM, Feeney P, Yanovski SZ, Bantle J, Montgomery B, et al. Suboptimal control of glycemia, blood pressure, and LDL cholesterol in overweight adults with diabetes: the Look AHEAD Study. J Diabetes Complicat 2008;22(1):1-9.

30. Chatterji P, Joo H, Lahiri K. Racial/ethnic- and education-related disparities in the control of risk factors for cardiovascular disease among individuals with diabetes. Diabetes Care 2012;35(2):305-12. 